\title{
Vortices in a Bose-Einstein Condensate
}

\author{
M. R. Matthews, B. P. Anderson, P. C. Haljan, D. S. Hall, C. E. Wieman, and E. A. Cornellt \\ JILA, National Institute of Standards and Technology and Department of Physics, \\ University of Colorado, Boulder, Colorado 80309-0440
}

(August 9, 1999)

We have created vortices in two-component Bose-Einstein condensates. The vortex state was created through a coherent process involving the spatial and temporal control of interconversion between the two components. Using an interference technique, we map the phase of the vortex state to confirm that it possesses angular momentum. We can create vortices in either of the two components and have observed differences in the dynamics and stability.

The concept of a vortex is at the center of our understanding of superfluidity. A vortex is a topological feature of a superfluid - in a closed path around the vortex, the phase undergoes a $2 \pi$ winding and the superfluid flow is quantized. Following the experimental realization of a dilute atomic Bose-Einstein condensate (BEC) [1], much theoretical effort has been directed towards the formation and behavior of vortices in atomic BEC [2]. This paper presents the experimental realization and imaging of a vortex in BEC. We use the method proposed by Williams and Holland [5] to create vortices in a two-component BEC. An interference technique is used to obtain phase images of the vortex state and confirm the $2 \pi$ phase winding required by the quantization condition. We have also carried out preliminary studies of the stability of the vortices.

Vortices are created in superfluid helium by cooling a rotating bucket of helium through the superfluid transition, and a vortex forms for each unit of angular momentum. This does not work for BEC because it is formed in a harmonic magnetic trap. When the condensate first forms it occupies a tiny cross-sectional area at the center of the trap and is too small to support a vortex. Eventually, the condensate grows to sufficient size that it can support vortices, but the time scale for vortices to be generated in the vortex-free condensate due to coupling with the rotating environment is unknown, and may well be longer than the lifetime of the condensate. This is the potential difficulty with using an optical "stirring beam" or magnetic field distortion to rotate the cloud during condensation, as has been frequently proposed. Another proposal has been to use optical beams with appropriate topologies to "imprint" a phase on an existing condensate. This technique must drive the local density to zero at some point and then rely on uncertain dissipative processes for the condensate to relax into a vortex state.
We have avoided these uncertainties by creating vortices using a coherent process that directly forms the desired vortex wave function via transitions between two internal spin states of ${ }^{87} \mathrm{Rb}$. The two spin states, henceforth $|1\rangle$ and $|2\rangle$, are separated by the ground-state hyperfine splitting and can be simultaneously confined in identical and fully overlapping magnetic trap potentials. A two-photon microwave field induces transitions between the states. As we have seen in previous experiments, this coupled two-component condensate is exempt from the topological rules governing single-component superfluids [6] - rules that make it difficult to implant a vortex within an existing condensate in a controlled manner. In the coupled system, we can directly create a $|2\rangle$ (or $|1\rangle)$ state wave function having a wide variety of shapes [5] out of a $|1\rangle$ (or $|2\rangle)$ ground-state wave function by controlling the spatial and temporal dependence of the microwave-induced conversion of $|1\rangle$ into $|2\rangle$.

We control the conversion by shifting the transition frequency using the AC Stark effect. A spatially inhomogeneous and moveable optical field (a focused laser beam) provides the desired spatial and temporal control of the AC Stark shift. The vortex state is an axially symmetric ring with a $2 \pi$ phase winding around the vortex core where the local density is zero. To create a wave function with this spatial symmetry, the laser beam is rotated around the initial condensate as in Fig. 1a. The desired spatial phase dependence is obtained by detuning the microwave frequency from the transition, and rotating the laser beam at the appropriate frequency $\omega$ to make the coupling resonant. For large microwave detunings $\delta$, the necessary rotation frequency is simply $\delta$. For smaller detunings, the rotation frequency must be the effective Rabi frequency of the microwave transition [7]. As shown in Fig. $1 \mathrm{~b}$ for large detunings, the energy resonance condition now means that atoms can only change internal state through the coupling of the time-varying perturbation, and are therefore obliged to obey any selection rule that the spatial symmetry of that perturbation might impose. The center of the condensate (at the axis of the beam rotation) feels no time-varying change, while regions near the circumference of the condensate feel a near-sinusoidal variation, with a phase delay equal to the azimuthal angle $\theta$ around the circumference of the cloud. Williams and Holland show that this is precisely the geometry best suited to couple the condensate into a vortex state. It should be emphasized that it is not simply the mechanical forces of the optical field that excite 
the vortex: a laser beam rotating clockwise can produce clockwise or counterclockwise vortex circulation, depending on the sign of the microwave detuning.

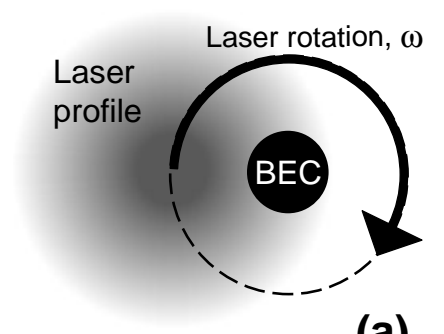

(a)

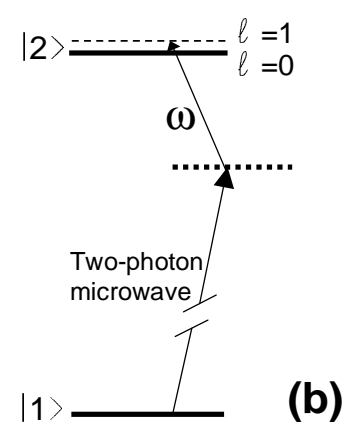

FIG. 1. (a) A basic schematic of the technique used to create a vortex. An off-resonant laser provides a rotating gradient in the AC Stark shift across the condensate as a microwave drive of detuning $\delta$ is applied. (b) A level diagram showing the microwave transition to very near the $|2\rangle$ state, and the modulation due to the laser rotation frequency that couples only to the angular momentum $l=1$ state when $\omega=\delta$. In the figure, the energy splitting $(<1 \mathrm{~Hz})$ between the $l=1$ and $l=0$ states is exaggerated.

In the absence of the microwave coupling field, the two states can be thought of as two distinguishable, interpenetrating superfluids that interact with each other and with themselves via a mean-field repulsion proportional to the local densities. The interaction coefficients differ slightly [8.9], so the $|1\rangle$ fluid has slight positive buoyancy with respect to the $|2\rangle$ fluid [10]. When the $|1\rangle$ fluid has a net angular momentum, it forms an equatorial ring around the central $|2\rangle$ fluid. The $|1\rangle$ fluid partially penetrates the constant-phase $|2\rangle$ fluid, which creates a central potential barrier. Conversely, a $|2\rangle$ vortex forms a ring that tends to contract down into the $|1\rangle$ fluid. We use the overlap of the $|1\rangle$ and $|2\rangle$ fluids to image the phase profile of the vortex state via the interconversion interference technique that we introduced in [11. In the presence of a near-resonant microwave field (and no perturbing optical field), the two states interconvert at a rate sensitive to the local difference in the quantum phases of the two states. Thus the application of a resonant $\pi / 2$ microwave pulse transforms the original two-fluid density distribution into a distribution that reflects the local phase difference, a "phase interferogram." Looking at the condensate both before and after the interconversion pulse provides images of both the amplitude and phase of the vortex ring.

The basic experimental setup for forming condensates and driving them between different spin states is the same as in [8]. Using laser cooling and trapping, followed by trapping in a TOP magnetic trap and evaporative cooling, we produce a condensate of typically $\sim 8 \times 10^{5}$ atoms in the $|1\rangle$ state $\left(F=1, m_{F}=-1\right)$. We then adiabati- caly convert the trap to a spherically symmetric potential by reducing the quadrupole magnetic field gradient [12]. This leaves us with a condensate 54 microns in diameter in a trap with oscillation frequencies of $7.8 \pm 0.1 \mathrm{~Hz}$ in the radial and axial directions for both spin states. In this trap, a $|1\rangle$ state condensate has a lifetime of $75 \mathrm{~s}$ and the $|2\rangle$ state $\left(F=2, m_{F}=+1\right)$ about $1 \mathrm{~s}$. Oscillating magnetic fields can then be pulsed on to drive the microwave transition between the $|1\rangle$ and $|2\rangle$ states. The power and oscillation frequency of the fields are adjusted to obtain the desired effective Rabi frequency for the $|1\rangle$ to $|2\rangle$ transition. To create a vortex in $|2\rangle$ we add a 10 $\mathrm{nW}, 780 \mathrm{~nm}$ laser beam that has a waist of $180 \mu \mathrm{m}$ and is detuned $0.8 \mathrm{GHz}$ blue of resonant excitation of the $|2\rangle$ state. Using piezoelectric actuators we rotate the beam in a $\sim 75 \mu \mathrm{m}$ radius circle around the condensate at 100 $\mathrm{Hz}$. The following procedure is used to obtain the precise location of the laser beam that is required to create a vortex. We first set the effective Rabi frequency to 100 $\mathrm{Hz}$ by adjusting the detuning of the microwave frequency away from resonance in the presence of the light. Typically the resonant Rabi frequency is $35 \mathrm{~Hz}$ and the total detuning about $94 \mathrm{~Hz}$ from the $|1\rangle$ to $|2\rangle$ transition. By making fine adjustments of the detuning we then optimize the amount of transfer (typically $50 \%$ ) to the $|2\rangle$ state. We then adjust the center of rotation of the beam to obtain the most symmetric rings. After $\sim 70 \mathrm{~ms}$ the vortex has been formed, and we turn off the laser beam and the microwave drive.

We can take multiple images of a vortex both during and after formation using nondestructive state-selective phase contrast imaging [6,13]. Rapid control of the microwave power and frequency allows us to apply various pulse sequences to explore many different options for the creation, manipulation, and observation of a single vortex. For example, we can put the initial condensate into either the $|1\rangle$ or $|2\rangle$ state and then make a vortex in the $|2\rangle$ or $|1\rangle$ state respectively, and we can obtain phase interferograms or quickly switch the internal state of the vortex at any time after the rotating laser beam is off. We can also watch the evolution of the vortex over time scales from milliseconds to seconds. All of these techniques are nondestructive to both the density and phase.

In Fig. 2 we show a detailed picture of the phase profile of a $|2\rangle$ vortex. To obtain this we first take a picture of the vortex (Fig. 2a), then we apply a resonant microwave $\pi$-pulse. Half-way through the $4 \mathrm{~ms}$ long $\pi$-pulse, we take a second image (Fig. 2b), and at the completion of the pulse we take a third image (Fig. 2 2c) that shows the original density distribution of the interior $|1\rangle$ state. Normalizing by the density distributions of the vortex and interior states we obtain the phase image in Fig. 2 $\mathrm{d}$ [14]. The figure dramatically shows the variation and continuity of the phase around the ring (Fig. 2 2 e) that are required by the quantization of angular momentum. 

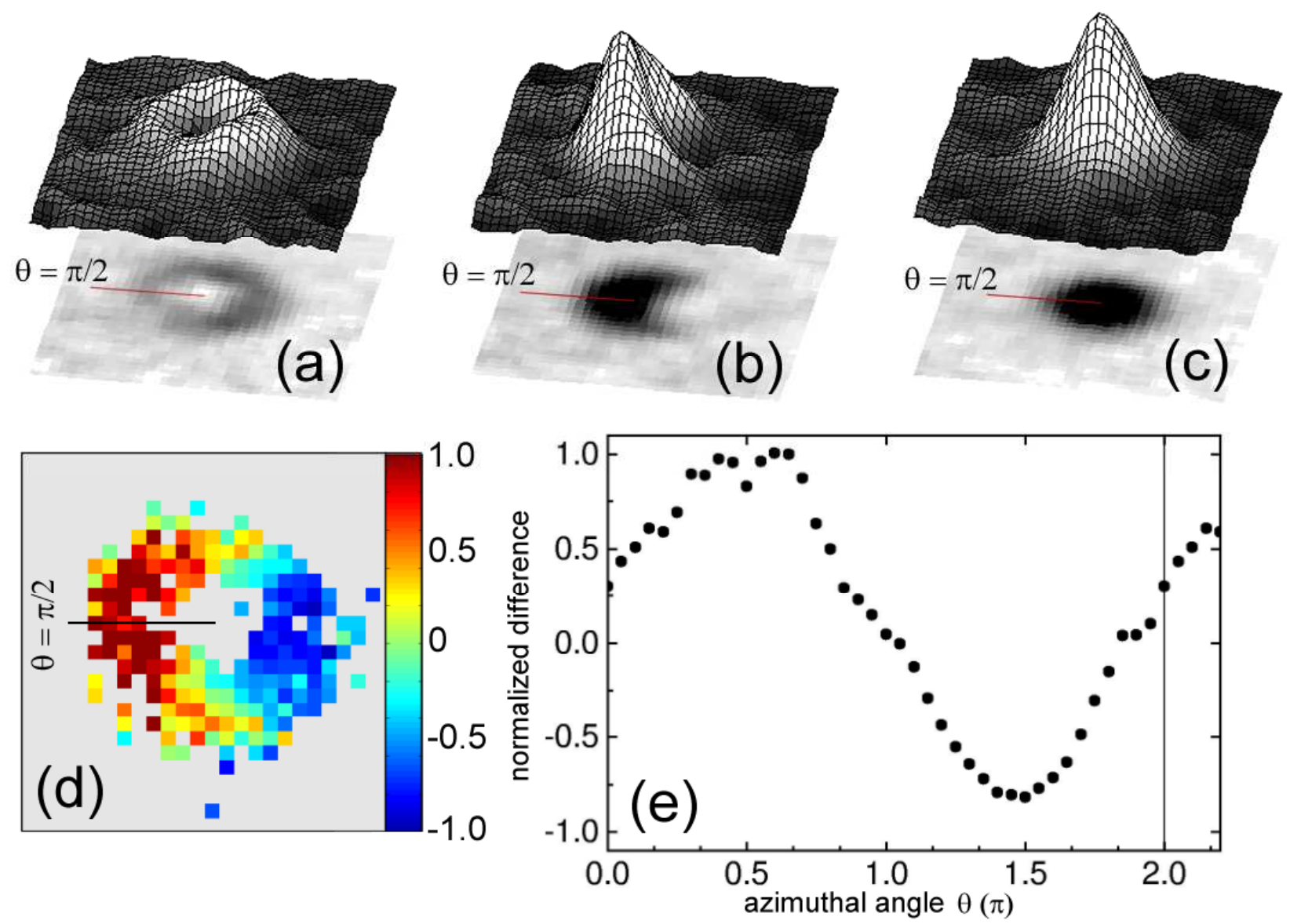

FIG. 2. Condensate images (100 $\mu \mathrm{m}$ on a side) in which the imaging laser is tuned such that only $|2\rangle$ is visible. (a) The intial image of the vortex, (b) after $\pi / 2$ of the interconversion pulse, and (c) after completion of the $\pi$-pulse. The vortex is now invisible (in $|1\rangle$ ) and the interior fluid is imaged (in $|2\rangle$ ). (d) The normalized difference in densities between the local average ring and interior densities ((a) and (c) respectively) and the phase interferogram density (b) for each corresponding point in the images. This is approximately the cosine of the local phase difference $\phi$ between the vortex state and the interior state [14]. The values are shown only for those regions where the densities of each state were high enough to give adequate signal to noise for the phase reconstruction. (e) The radial average at each angle $\theta$ around the ring is shown in (d). (The data is repeated after the azimuthal angle $2 \pi$ to better show the continuity around the ring.) 
In Fig. 3 are pictures of the time evolution of vortices to show their dynamics and stability. As expected, the dynamics of the $|1\rangle$ state vortices (Fig. $3 a$ and Fig. $3 \mathrm{~b}$ ) are different from the $|2\rangle$ state vortices (Fig. 3ic) due to the different scattering lengths. The $|2\rangle$ vortex ring sinks in toward the trap center (Fig. 3c), and then rebounds and apparently breaks up. This pattern is repeatedly observed in measurements of the $|2\rangle$ vortices. Conversely, the equilibrium position of a $|1\rangle$ state fluid is obtained by "floating" outside the $|2\rangle$ state fluid. The inner radius of the $|1\rangle$ state ring shrinks slowly as the interior $|2\rangle$ fluid decays away with a $\sim 1 \mathrm{~s}$ lifetime. A variety of additional behaviors has been seen for $|1\rangle$ vortices. Initial asymmetry is very sensitive to beam position and condensate slosh. For small differences in the initial vortex state density distribution, asymmetric density distributions sometimes develop and/or "heal" between 0.5 and $1 \mathrm{~s}$.

The nonrotating $|2\rangle$ fluid within a $|1\rangle$ vortex is analogous to the defects that pin vortices in superconductors. This $|2\rangle$ "defect" can be removed quickly, by a properly tuned laser pulse, or allowed to decay slowly (as in Figs. 3a and 3b). Thus this system can be varied between two relevant physical limits. In the limit of a large repulsive central potential (produced by a large amount of $|2\rangle$ fluid) the system most closely resembles quantized flow in a fixed, 3-D toroidal potential. The vortex core is pinned and its size is determined by the central potential. Figs. $3 \mathrm{a}$ and $3 \mathrm{~b}$ are near this limit for $t=0$ and $200 \mathrm{~ms}$. In this case the density distribution of the vortex state is vulnerable to instabilities due to the fact that the relative densities of $|1\rangle$ and $|2\rangle$ may evolve with relatively little energy cost so long as the total density remains constant [10]. In the opposite limit (a small amount of $|2\rangle$ fluid), the central potential is negligible and the size of the vortex core (in equilibrium) is determined entirely by its own centrifugal barrier. Figs. $3 \mathrm{a}$ and $3 \mathrm{~b}$ at $t=600 \mathrm{~ms}$ are evolving towards this limit. In Fig. Ba, we see that the interior $|2\rangle$ fluid is no longer pinning the vortex at 600 $\mathrm{ms}$, but rather is being dragged around by the precessing vortex. The $|2\rangle$ vortex provides an interesting mixture of the above limits. The $|1\rangle$ fluid "floats" to the outside so there is no pinning of the vortex core, but the tendency towards density instabilities remains.

Expanded studies of stability issues are underway. We also expect to be able to observe interesting transitional behavior between these limiting cases. For example, it is a straightforward extension of our method to create an $l=2$ vortex. In the presence of a strong pinning potential, $l=2$ vortices should be stable, but in the weak potential limit, $l=2$ vortices are predicted to spontaneously bifurcate [4]. We gratefully acknowledge useful conversations with J. Williams and M. Holland. This work is supported by the ONR, NSF, and NIST.

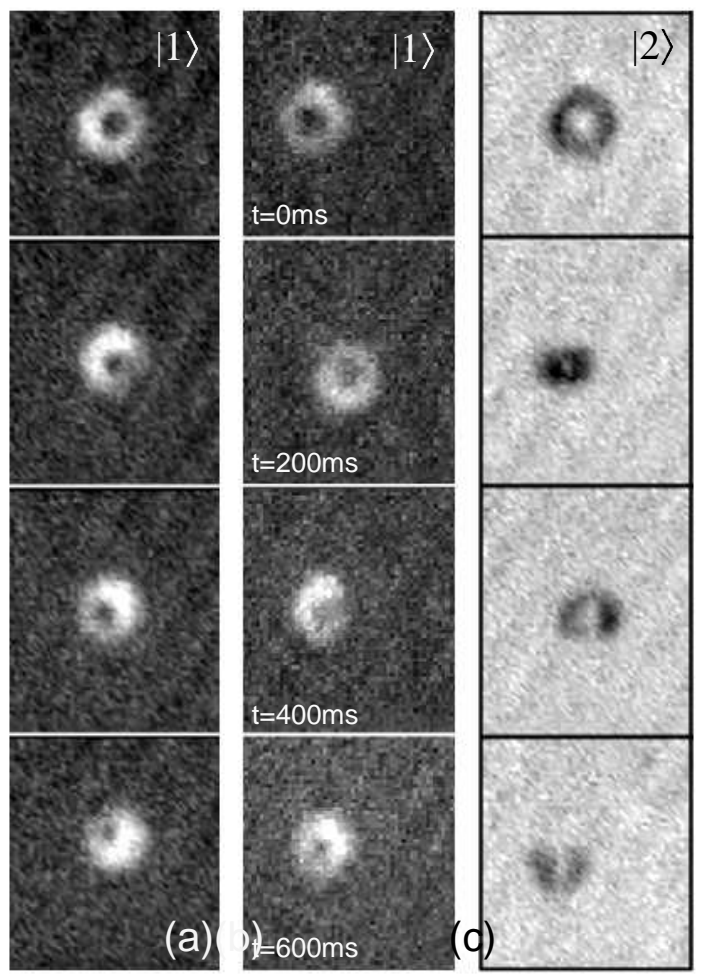

FIG. 3. (a,b) Two separate instances of the free evolution of a $|1\rangle$ state vortex in the magnetic trap. It is stable over a time long compared to the trap oscillation period (128 ms). (c) The free evolution of a $|2\rangle$ vortex is much more dynamic. It is seen shrinking quickly into the invisible $|1\rangle$ fluid and rebounding into fragments. Each column is from a single run, where time $t$ is referenced to the end of vortex creation ( $t$ is the same for each row). The $|1\rangle$ and $|2\rangle$ state images appear different due to different signs of the probe detuning.

* Quantum Physics Division, National Institute of Standards and Technology.

$\dagger$ Present address: Department of Physics, Amherst College, Amherst, MA 01002.

[1] M. H. Anderson et al., Science 269, 198 (1995); W. Ketterle et al., cond-mat/9904034; E. Cornell et al., condmat/9903109.

[2] K.-P. Marzlin, W. Zhang, and E. M. Wright, Phys. Rev. Lett. 79, 4728 (1997); R. Dum, J. I. Cirac, M. Lewenstein, and P. Zoller, Phys. Rev. Lett. 80, 2972 (1998); E. L. Bolda and D. F. Walls, Phys. Lett. A 246, 32 (1998). These vortex generation methods involve transitions between different internal states, as in reference [5].

[3] F. Dalfovo and S. Stringari, Phys. Rev. A 53, 2477 (1996); T.-L. Ho and V. B. Shenoy, Phys. Rev. Lett. 77, 2595 (1996); D. S. Rokhsar, Phys. Rev. Lett. 79, 2164 (1997); R. Dodd, K. Burnett, M. Edwards, and C. 
Clark, Phys. Rev. A 56, 587 (1997); B. Jackson, J. F. McCann, and C. S. Adams, Phys. Rev. Lett. 80, 3903 (1998); A. Fetter, Journal Low. Temp. Phys. 113, 189 (1998); S. Stringari, Phys. Rev. Lett. 82, 4371 (1999); T. Isoshima and K. Machida, Phys. Rev. A 59, 2203 (1999); B. Caradoc-Davies, R. Ballagh, and K. Burnett, Phys. Rev. Lett. 83, 895 (1999); D. Feder, C. Clark, and B. Schneider, Phys. Rev. Lett. 82, 4956 (1999).

[4] H. Pu, C. Law, J. Eberly, and N. Bigelow, Phys. Rev. A 59, 1533 (1999).

[5] J. Williams and M. Holland, submitted to Nature, (1999).

[6] M. R. Matthews et al., cond-mat/9906288.

[7] In the limit of large detuning, $\delta$ is equal to the effective Rabi frequency. In our implementation, $\omega$ and the effective Rabi frequency are $100 \mathrm{~Hz}$ and $\delta$ is $94 \mathrm{~Hz}$.

[8] M. R. Matthews et al., Phys. Rev. Lett. 81, 243 (1998).

[9] J. P. Burke, Jr., J. L. Bohn, B. D. Esry, and C. H. Greene, Phys. Rev. Lett. 80, 2097 (1998).

[10] D. S. Hall et al., Phys. Rev. Lett. 81, 1539 (1998).

[11] D. S. Hall, M. R. Matthews, C. E. Wieman, and E. A. Cornell, Phys. Rev. Lett. 81, 1543 (1998).

[12] J.R. Ensher PhD. thesis, University of Colorado, 1998.

[13] M. R. Andrews et al., Science 273, 84 (1996).

[14] The normalized difference is defined as $\left(2 d_{|1\rangle|2\rangle}-d_{|2\rangle}-\right.$ $\left.d_{|1\rangle}\right) /\left(2 \sqrt{d_{|1\rangle} d_{|2\rangle}}\right)$ where $d_{|i\rangle}$ is the local density for the $i$ th state and $d_{|1\rangle|2\rangle}$ for the interferogram (Fig. $2 \mathrm{~b}$ ). This is not exactly the cosine of the phase difference $\phi$ between the vortex and interior state due to small effects, such as incomplete overlap between the states along the line of sight and uncertainties in the zero levels. 